\title{
Peripheral T-Cell Lymphoma of Cervical Spine
}

\author{
Foot-Juh Lian ${ }^{1}$, Wen-Chiuan Tsai ${ }^{2}$, Cheng-Ta Hsieh ${ }^{1^{*}}$ \\ ${ }^{1}$ Division of Neurosurgery, Department of Surgery, Cathay General Hospital, Taipei, Taiwan (China) \\ ${ }^{2}$ Department of Pathology, Tri-Service General Hospital, National Defense Medical Center, Taipei, Taiwan (China) \\ Email: "nogor@mail2000.com.tw
}

Received November 1, 2011; revised December 28, 2011; accepted January 10, 2012

\begin{abstract}
Peripheral T-cell lymphoma is a rather uncommon non-Hodgkin's lymphoma with the initial manifestation of spinal cord compression. Herein, we reported a 74-year-old woman with sustained neck pain radiating into the right shoulder and arm and weakness of the right upper extremity. A mass that had invaded the C5 and C6 vertebral bodies, causing a kyphotic curvature and compressing the spinal cord, was discovered with magnetic resonance imaging. The patient then underwent anterior corpectomy at C5 and C6, and reconstruction with a titanic rod and bone cement. The pathology confirmed a diagnosis of peripheral T-cell lymphoma after serial $\mathrm{H} \& \mathrm{E}$ and immunohistochemical staining. She recovered well from her profound neurological deficit. Both chemotherapy and radiotherapy were used postoperatively. Surgical intervention is indicated in these cases to decompress the cord, remove the majority of the tumor mass, stabilize the spine and obtain tissue for pathological diagnosis.
\end{abstract}

Keywords: Spinal Cord Compression; Vertebral Neoplasm; Cervical Spine; Peripheral T-Cell Lymphoma

\section{Introduction}

Neck and radicular pain are frequent complications and may result from conditions such as herniated intervertebral discs, overgrowth of bone intruding into the spinal canal, spinal stenosis, fracture-dislocation of the spine, infection, or neoplasms [1]. However, spinal cord compression as the first presentation of peripheral T-cell lymphomas (PTCLs) has been rarely reported in the literature [2]. Our objective in presenting this particular case is to highlight the rare presentation of peripheral T-cell lymphoma involving the cervical spine.

\section{Case Report}

A 74-year-old previously healthy woman complained of neck pain radiating into her right shoulder and arm and weakness of the right upper extremity for nearly 6 months prior to admission. Physical examinations showed no remarkable findings except a mild decrease in muscle power (strength score 4/5) in the right shoulder and arm. Lymph node enlargement at the right neck and bilateral inguinal regions was palpable. Laboratory examinations did not provide additional diagnostic information. Radiographs of the cervical spine revealed a kyphotic curvature, deformity of the $\mathrm{C} 4$ and $\mathrm{C} 5$ vertebral bodies, and narrowed disc spaces between C4-5, C5-6 and C6-7. Magnetic resonance imaging of the cervical spine revealed a solitary tumor

${ }^{*}$ Corresponding author. expanding from $\mathrm{C} 2$ to $\mathrm{C} 7$, particularly with destruction of the C5 and C6 vertebral bodies and compression of the spinal cord (Figures 1(a) and 1(b)). In addition, encasement of the carotid artery and internal jugular vein by the tumor was also observed. The patient underwent anterior corpectomy of C5 and C6 with reconstruction using a titanium rod and bone cement. On operation, the soft and yellowish-brown mass was found to have invaded C5 and C6 from the prevertebral through the right foramen region, with compression of the nerve roots and spinal cord. Diffuse small to medium-sized tumor cells with an irregular nuclear contour, inconspicuous nucleoli, scanty cytoplasm, and occasional mitosis were observed microscopically (Figure 2(a)). Immunobiochemical staining was diffusely positive for CD45 and CD3 antigens (Figures 2(b) and 2(c)), with focal expression of vimentin, and negative for CK, CD20 and chromogranin A. Based on the above findings, the pathological report confirmed a diagnosis of PTCL. CEOP therapy (cyclophosphamide, epirubicin, vincristine, and prednisolone) and local radiotherapy were administrated postoperatively and the nodal lesions disappeared. She recovered well from her profound neurological deficits.

\section{Discussion}

PTCLs, derived from mature post-thymic lymphocytes, are rather uncommon non-Hodgkin's lymphoma (NHLs) and the prevalence depends on the country [3]; in Western 


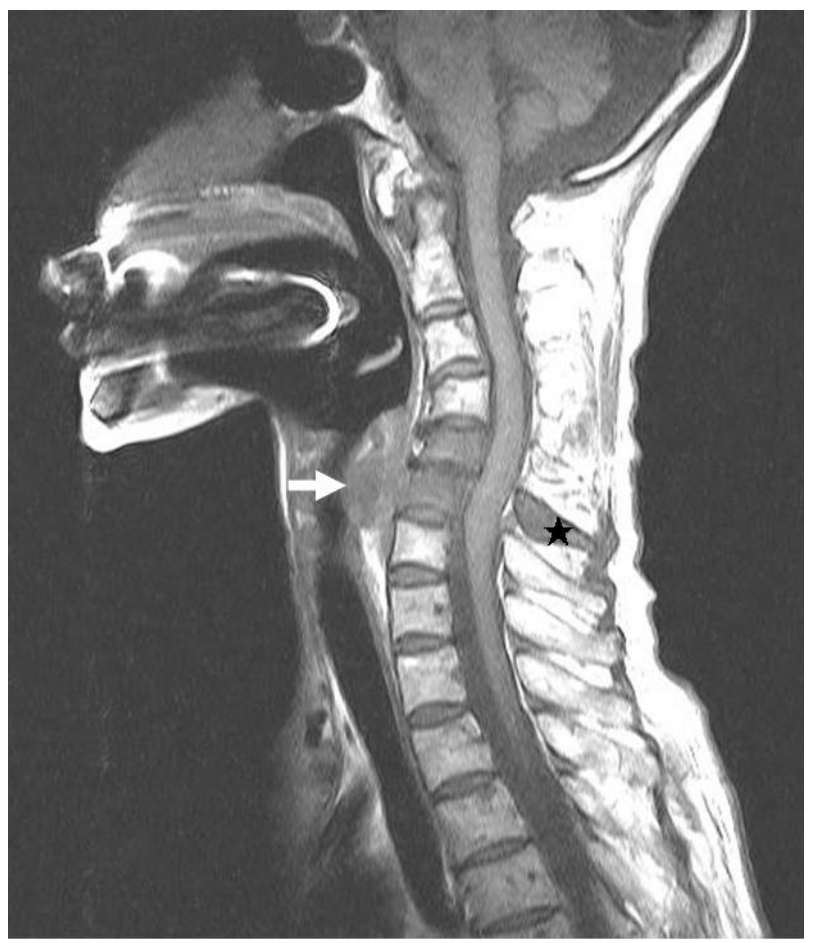

(a)

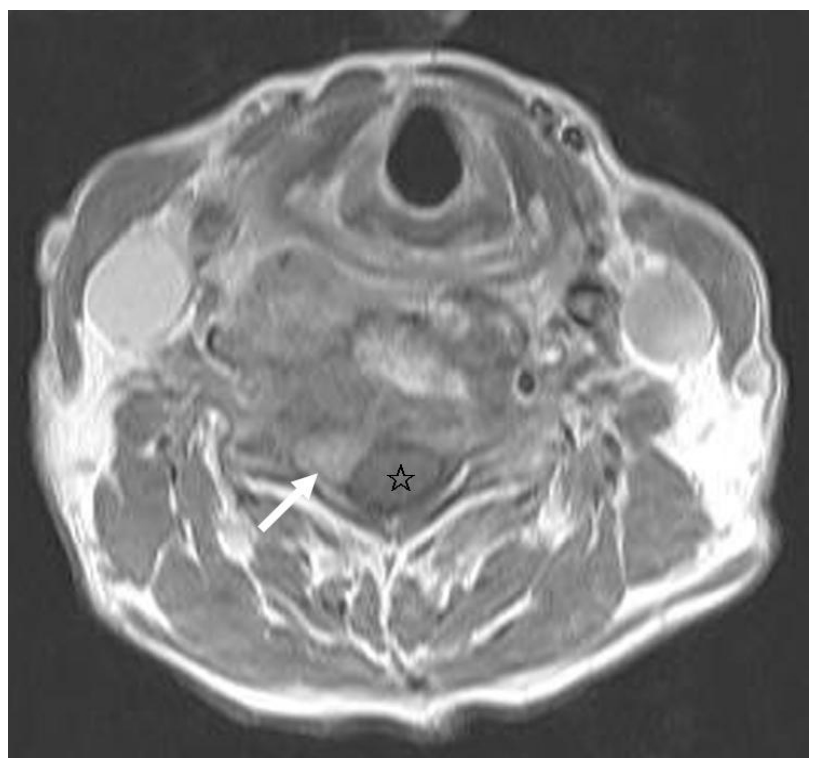

(b)

Figure 1. Magnetic resonance imaging of the cervical spine. (a) Sagittal view of T1-weighted images revealed the mass (white arrow) had invaded the C5 and C6 vertebral bodies, from the prevertebral to the spinal process (black asterisk); (b) Axial view of T1-weighted images revealed the vertebral mass (white arrow) with compression of the spinal cord (asterisk).

countries, PTCLs account for $15 \%$ to $20 \%$ of aggressive lymphomas and $7 \%$ to $10 \%$ of all NHLs [4]. This proportion increases to approximately $25 \%$ in Korea and $70 \%$ to $80 \%$ in Japan for epidemiological reasons such as

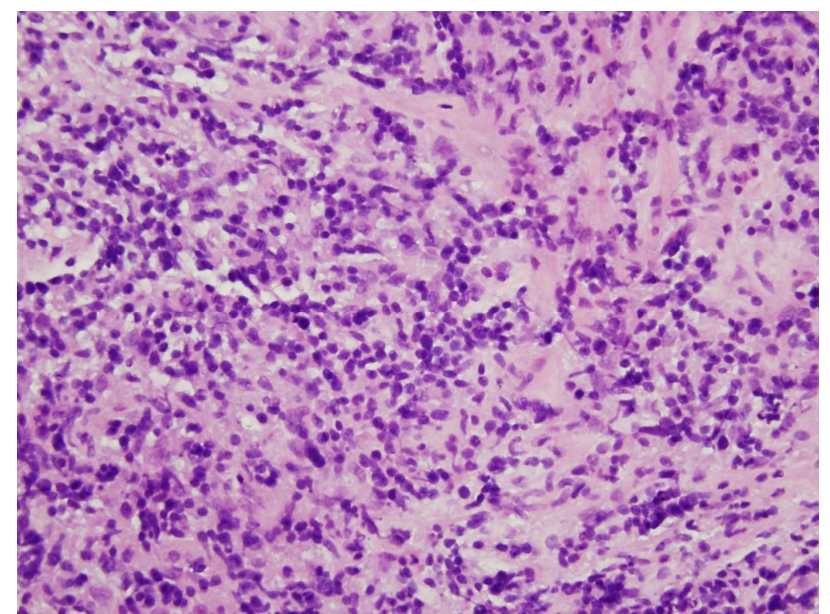

(a)

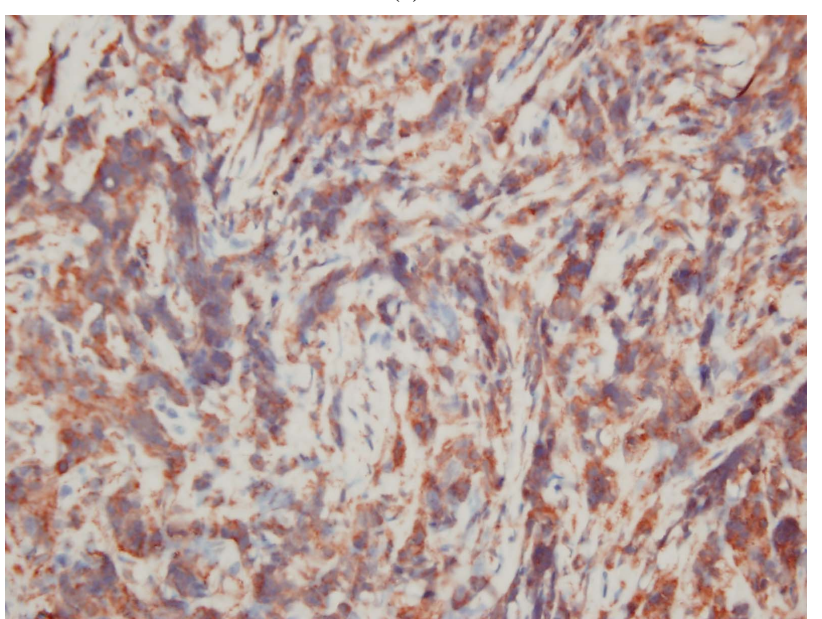

(b)

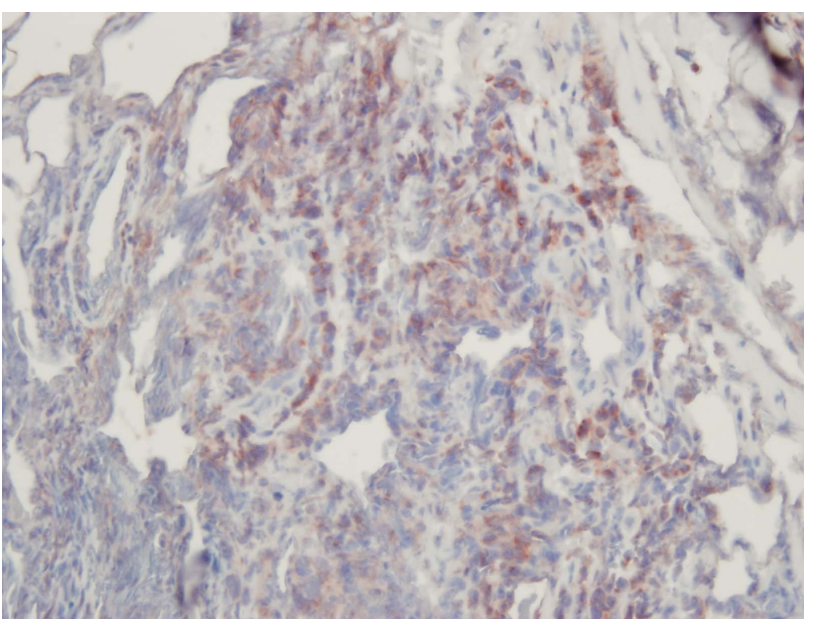

(c)

Figure 2. Pathological images. (a) High-power view showed diffusely small to medium-sized tumor cells arranged in a diffuse pattern. (H \& E ×400); (b) The tumor cells were immunoreactive for CD45 (C, $\times 400)$; (c) The tumor cells were immunoreactive for CD3 $(\mathrm{C}, \times 400)$.

human T-lymphotropic virus 1 infection [5,6]. Although 
PTCLs have been reported in all groups, including young individuals, it generally affects the elderly, with a median age in the 5th to 6th decade and an equal sex distribution [7]. Although involvement of the sinonasal cavity, airway, intestinal tract, skin, lymph nodes, lung, liver, spleen, and musculoskeletal system has been reported, the central nervous system, especially the spine, is rarely involved $[5,8]$. In a series of 453 previously untreated NHL patients seen at the Northern Israel Oncology Center between 1968 and 1983, Epelbaum et al. reported only 10 patients (2.2\%) presenting with spinal cord compression [9]. The overall occurrence rate was $0.1 \%$ to $6.5 \%$ among people with non-Hodgkin's lymphoma [10].

A predilection for involvement of the thoracic and lumbar region has been reported in patients with NHLs involving the spine [9]. The spinal extradural space is the most common site, because of an isolated deposit within the spinal canal or by extension from an adjacent nodal mass or bone involvement [1]. Spinal cord compression may result from the tumor tissue extending from the paraspinal lymph nodes through the intervertebral foramina into the epidural space or expanding from the involved vertebral bodies [11]. Clinical presentations vary from asymptomatic to paralysis, and are expected with any epidural tumor of the spinal canal [12]. Two phases, including the prodromal phase (local and radicular pain persisting for several months to 1 year) and the spinal cord compression phase (rapidly developing signs of compression within 2 to 8 weeks), have been hypothesized as the clinical course of spinal metastasis [9]. The interval between the initial symptoms of back pain and the first evidence of vertebral or epidural lesion has been figured from 7 to 180 days, with a mean of 58 days [13].

Although plain radiograph of the spine is still the first choice to rule out bony destruction or vertebral fractures, MRI has a better effect in differentiating these spinal diseases, especially soft tissues such as neoplasms, the spinal cord and intervertebral discs [1,9]. However, like our case, the differential diagnosis of an extradural mass with vertebral involvement includes metastases (most common), primary neoplasms, and infectious spondylodiscitis with epidural extension. In vertebral metastases, most original tumors are from the breast (22\%), lung (15\%), prostate (10\%), lymphoma (10\%), sarcoma (9\%), kidney (7\%), and gastrointestinal tract (5\%) [14]. A detailed history, accurate physical examinations, and laboratory examinations are essential for the first clinical diagnosis. Decompressive surgery is strictly indicated if there are severe neurological deficits or rapidly progressing neurological signs, as in our case [8]. Percutaneous transpedicular biopsy is an alternative diagnostic method if there are no symptoms or signs of spinal cord compression.

Most patients with NHLs are treated with chemotherapy and radiotherapy. Compared with similar histological grades of B-cell lymphoma, the clinical course of PTCL is usually aggressive and prone to relapse [5]; the majority of PTCLs have a poorer response to chemotherapy than B-cell lymphomas [3,6]. The 5-year survival rate in patients with PTCL with chemotherapy ranged from $25 \%$ to $47 \%$ in the literature [15]. Combination chemotherapy and radiography has been proved to improve the survival rate $[7,9,15]$. Survival was significantly associated with the International Prognostic Factor Index, such as the stage of the disease, performance status, and pretreatment LDH value [16]. However, the prognosis for central nervous system lymphoma without treatment is poor, and median survival has been estimated to be about 4.6 months only [17].

\section{Conclusions}

In conclusion, spinal cord compression could be the initial presentation of peripheral T-cell lymphoma. A detailed history, physical examination, laboratory studies and radiological screening are essential to differentiate spinal diseases. Surgery is indicated to decompress the cord, remove the majority of the tumor mass, stabilize the spine and obtain tissue for pathological diagnosis.

\section{REFERENCES}

[1] B. Vanneuville, A. Janssens, M. Lemmerling, K. de Vlam, H. Mielants and E. M. Veys, "Non-Hodgkin's Lymphoma Presenting with Spinal Involvement," Annals of the Rheumatic Diseases, Vol. 59, No. 1, 2000, pp. 12-14. doi:10.1136/ard.59.1.12

[2] J. R. Perry, S. S. Deodhare, J. M. Bilbao, D. Murray and P. Muller, "The Significance of Spinal Cord Compression as the Initial Manifestation of Lymphoma,” Neurosurgery, Vol. 32, No. 2, 1993, pp. 157-162. doi:10.1227/00006123-199302000-00001

[3] C. E. Dearden and F. M. Foss, "Peripheral T-Cell Lymphomas: Diagnosis and Management," Hematology/Oncology Clinics of North America, Vol. 17, No. 6, 2003, pp. 1351-1366. doi:10.1016/S0889-8588(03)00119-9

[4] A. Gallamini, C. Stelitano, R. Calvi, M. Bellei, D. Mattei and U. Vitolo, "Peripheral T-Cell Lymphoma Unspecified (PTCL-U): A New Prognostic Model from a Retrospective Multicentric Clinical Study,” Blood, Vol. 103, No. 7, 2004, pp. 2474-2479. doi:10.1182/blood-2003-09-3080

[5] H. J. Lee, J. G. Im, J. M. Goo, K. W. Kim, B. I. Choi and K. H. Chang, "Peripheral T-Cell Lymphoma: Spectrum of Imaging Findings with Clinical and Pathologic Features,” Radiographics, Vol. 23, No. 1, 2003, pp. 7-26. doi:10.1148/rg.231025018

[6] A. Lopez-Guillermo, J. Cid, A. Salar, A. Lopez, C. Montalban and J. M. Castrillo, "Peripheral T-Cell Lymphomas: Initial Features, Natural History, and Prognostic Factors in a Series of 174 Patients Diagnosed According to the R.E.A.L. Classification,” Annals of Oncology, Vol. 9, No. 8, 1998, pp. 849-855. doi:10.1023/A:1008418727472 
[7] Z. Klezl, M. Krbec, E. Gregora and J. Stritesky, "Rare Presentation of Non-Hodgkin Lymphoma of the Thoracolumbar Spine in Pregnancy with 7 Years' Survival," Archives of Orthopaedic and Trauma Surgery, Vol. 122, No. 5, 2002, pp. 308-310.

[8] G. S. Pinkus, C. J. O’ara and J. W. Said, “Eripheral/PostThymic T-Cell Lymphomas: A Spectrum of Disease. Clinical, Pathogic, and Immunologic Features of 78 Cases,” Cancer, Vol. 65, No. 4, 1990, pp. 971-998. doi:10.1002/1097-0142(19900215)65:4<971::AID-CNCR 2820650425>3.0.CO;2-B

[9] R. Epelbaum, N. Haim, M. Ben-Shahar, Y. Ben-Arie, M. Feinsod and Y. Cohen, “On-Hodgkin' Lymphoma Presenting with Spinal Epidural Involvement," Cancer, Vol. 58, No. 9, 1986, pp. 2120-2124. doi:10.1002/1097-0142(19861101)58:9<2120::AID-CNC R2820580926>3.0.CO;2-A

[10] C. C. Luo, "Spinal Cord Compression Secondary to Metastatic Non-Hodgkins Lymphoma: A Case Report,” Archives of Physical Medicine and Rehabilitation, Vol. 86, No. 2, 2005, pp. 332-334. doi:10.1016/j.apmr.2004.06.063

[11] J. W. Grant, D. Kaech and D. B. Jones, "Spinal Cord Compression as the First Presentation of Lymphoma-A Review of 15 Cases," Histopathology, Vol. 10, No. 11, 1986, pp. 1191-1202. doi:10.1111/j.1365-2559.1986.tb02559.x
[12] E. T. Wong, C. S. Portlock, J. P. O’Brien and L. M. DeAngelis, "Chemosensitive Epidural Spinal Cord Disease in Non-Hodgkins Lymphoma,” Neurology, Vol. 46, No. 6, 1996, pp. 1543-1547.

[13] J. Correale, D. A. Monteverde, J. A. Bueri and E. G. Reich, "Peripheral Nervous System and Spinal Cord Involvement in Lymphoma," Acta Neurologica Scandinavica, Vol. 83, No. 1, 1991, pp. 45-51. doi:10.1111/j.1600-0404.1991.tb03957.x

[14] C. Andreula and M. Murrone, "Metastatic Disease of the Spine,” European Radiology, Vol. 15, No. 3, 2005, pp. 627-632. doi:10.1007/s00330-004-2627-3

[15] Y. Huang, T. Y. Lin, Q. L. Wu, Z. L. Su, H. Q. Huang and Z. J. Xia, "Survival Outcomes of T-Cell Non-Hodgkin’s Lymphoma: A Report of 111 Cases,” Ai Zheng, Vol. 24, No. 4, 2005, pp. 470-474.

[16] S. M. Ansell, T. M. Habermann, P. J. Kurtin, T. E. Witzig, M. G. Chen and C. Y. Li, "Predictive Capacity of the International Prognostic Factor Index in Patients with Peripheral T-Cell Lymphoma,” Journal of Clinical Oncology, Vol. 15, No. 6, 1997, pp. 2296-2301.

[17] R. Viswanathan, N. K. Swamy, J. Vago and S. B. Dunsker, "Lymphoma of the Lumbar Nerve Root: Case Report,” Neurosurgery, Vol. 41, No. 2, 1997, pp. 479-481. doi:10.1097/00006123-199708000-00033 